\title{
Nonlinear dynamics of a planar hinged-supported beam with one end spring system
}

\author{
Łukasz Kłoda ${ }^{1,2, *}$, Stefano Lenci ${ }^{1}$ and Jerzy Warmiński ${ }^{2}$ \\ ${ }^{1}$ Department of Civil and Buildings Engineering, and Architecture, Polytechnic University of Marche, Ancona, Italy \\ ${ }^{2}$ Department of Applied Mechanics, Lublin University of Technology, Lublin, Poland
}

\begin{abstract}
Forced nonlinear oscillations of a planar, initially straight, Timoshenko beam are studied. The goal of this paper is to examine large amplitude vibrations of a hinged-simply supported beam with a linear axial spring on one end, allowing to consider the influence of varying axial boundary conditions. Influence of geometrical nonlinearity coming from very large deformations in axial, transversal and rotational directions on frequency response curves are presented for a wide spectrum of the spring stiffness. The complete frequency response curve is computed by a special pseudo continuation method of explicit numerical simulations. For selected cases, a jump phenomenon between branches and super/sub harmonic resonances have been observed.
\end{abstract}

\section{Introduction}

Large beam vibrations of a planar initially straight beam, where shear deformation and rotational bending effects are taken into account are presented e.g. in [1]. The nonplanar, nonlinear and inextensional beam which is undergoing flexure about two principal axes and torsion around longitudinal axis is studied in [2,3]. The beam is modeled as a set of mathematically consistent partial differential equations in [2], and its resonant forced oscillations are studied in [3]. However, those papers discussed only transversal and shear vibrations. In [4] the model of hinged beam is extended to take into account also longitudinal and rotatory inertia. Using the Galerkin method of modal expansion with arbitrary boundary conditions, a system of coupled nonlinear ordinary differential equations are first obtained and then investigated.

Lenci, Rega and Clementi [5-8] developed the exact equations of motion of a planar, initially straight beam, by considering axial, bending and shear deformations, and axial, transversal and rotatory inertia. It allowed to study the influence of longitudinal vibrations on dynamics of hinged-movable supported beam with axial linear spring on its end. The works [5-8] are focused on free nonlinear oscillations considering various spring stiffnesses. Using the Poincarè-Lindstedt method [5], the effect of variable slenderness [6] and axial-transversal coupling vibrations [7] has been obtained analytically and then validated numerically in [8].

The goal of this paper is to study forced and damped oscillations numerically by the Finite Element Method (FEM), using to commercial Abaqus_CAE® software. The FEM is applied by a special technique, similar to continuation method. In particular, for fixed values of the harmonic excitation amplitude, the frequency response curves are obtained by continuously varying the excitation frequency.

\section{The beam model}

Let us consider the Timoshenko beam presented in Fig. 1. It has length $L$, is straight in the initial configuration and has prismatic cross section $A$ with homogeneous material properties: Young modulus $E$, shear modulus $G$ and density $\rho$. The system is hingedsimply supported with one end axial spring with stiffness $\kappa$ in the moving end, see Fig. 1. Displacements in the axial and the transversal directions are denoted by $W(Z, T)$ and $U(Z, T)$, respectively, where $Z$ is a spatial coordinate and $T$ is time.

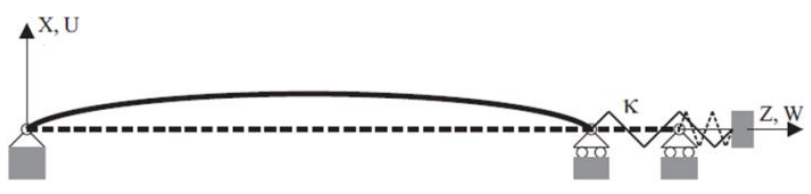

Fig. 1. Deformed configuration of the initially straight beam with one end linear spring.

Detailed description of kinematics, constitutive elastic behavior, boundary conditions and the derivation of Partial Differential Equations (PDEs) of motion are given in [5]. In this paper we extend the mathematical model presented in [5] adding harmonic excitation and linear viscous damping, as shown in Fig. 2. The equations of forces and moments balance for horizontal (1), vertical (2) motions and rotation (3) are:

\footnotetext{
* Corresponding author: 1.kloda@pollub.pl
} 
a)

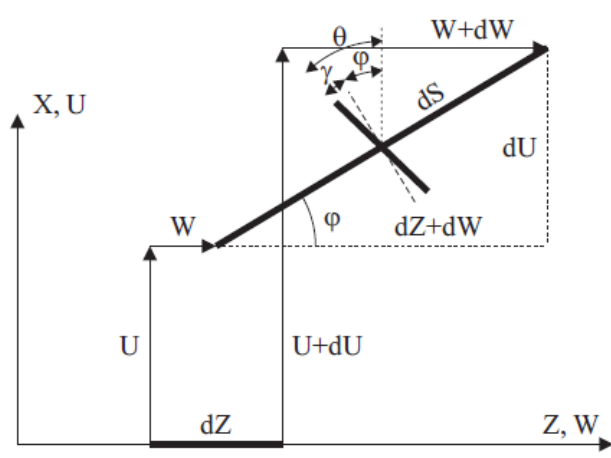

b)

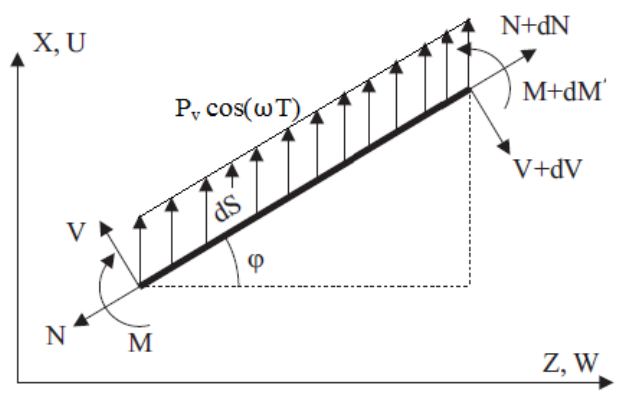

Fig. 2. The beam element deformations and rotation (a): $d Z-$ infinitesimal element in its initial configuration, $\mathrm{d} S$ - deformed element, $\mathrm{d} U, \mathrm{~d} W$-incremental displacements, $\theta$-rotation of the beam cross-section, $\varphi$ - slope angle of beam axis, $\gamma$ - shear strain and (b) balance of forces and moments: $\mathrm{N}$ - axial force, $\mathrm{V}$ - shear force, $\mathrm{M}$ - bending moment, $P_{v}-X$-directional uniformly distributed external harmonic excitation of frequency $\omega$.

$$
\begin{gathered}
(N \cos \varphi+V \sin \varphi)^{\prime}=\rho A \ddot{W}+2 C_{h} \dot{W}, \\
(N \sin \varphi-V \cos \varphi)^{\prime}= \\
\rho A \ddot{U}+2 C_{v} \dot{U}+P_{v} \cos (\omega T), \\
M^{\prime}-V S^{\prime}=\rho J \ddot{\theta}+2 C_{r} \dot{\theta} .
\end{gathered}
$$

In Eqs.(1)-(3) the dot corresponds to the derivative with respect to time $T$ and the prime denotes the spatial derivative with respect to axial coordinate $Z . C_{h}, C_{v}$ and $C_{r}$ are linear viscous damping factors in horizontal, vertical and rotational directions, respectively. Formulas (1), (2), (3) lead to the exact forced-damped PDEs of motion without approximations or condensation.

The boundary conditions are:

$$
\begin{gathered}
U(0, T)=U(L, T)=0, \\
M(0, T)=M(L, T)=0, \\
W(0, T)=0,
\end{gathered}
$$

while the boundary conditions provided by the axial spring in horizontal direction has the form:

$$
[N(L, T) \cos \varphi+V(L, T) \sin \varphi]+k_{s} W(L, T)=0 .
$$

Note that for $\kappa=\frac{k_{S} L}{E A}=0$ we get simply supported beam. When $\kappa$ tends to $\infty$ it implies $W(L, T)=0$ and the beam becomes hinged-hinged. Other cases of $\kappa$ represent hinged-simply supported-spring systems.

\section{Finite Element Model}

The problem is addressed by the FEM, where we consider classic Timoshenko beam with length to width ratio equal to 10 . Beam has been discretized by using 100 finite elements of B31 type. This element type has linear shape between nodes, in three-dimensional space.

\begin{tabular}{|c|c|c|}
\hline \multicolumn{2}{|c|}{ Initial length $[L]$} & $500 \mathrm{~mm}$ \\
\hline \multicolumn{2}{|c|}{ Cross section $[A]$} & $50 \mathrm{~mm} \times 50 \mathrm{~mm}$ \\
\hline \multicolumn{2}{|c|}{ Density $[\rho]$} & $7850 \mathrm{~kg} / \mathrm{m}^{3}$ \\
\hline \multicolumn{2}{|c|}{ Young modulus $[E]$} & $210 \mathrm{Gpa}$ \\
\hline \multicolumn{2}{|c|}{ Poisson's ratio $[v]$} & 0.3 \\
\hline \multicolumn{2}{|c|}{ Beams shear factor $[\mathrm{Sk}]$} & 0.85 \\
\hline \multicolumn{2}{|c|}{ Viscous damping factor $[\zeta]$} & $6 \%$ \\
\hline \multirow{3}{*}{ 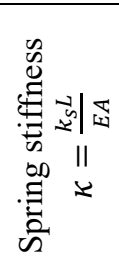 } & $\begin{array}{l}\text { Hinged- simply } \\
\text { supported beam }\end{array}$ & $\kappa=0$ \\
\hline & $\begin{array}{c}\text { Hinged-simply } \\
\text { supported-axial } \\
\text { spring beam system }\end{array}$ & $0<\kappa<\infty$ \\
\hline & Hinged-hinged & $\kappa=\infty$ \\
\hline \multicolumn{2}{|c|}{$\begin{array}{l}\text { External excitation amplitude } \\
\qquad\left[p_{v}\right]\end{array}$} & $40.7992 \mathrm{kN}$ \\
\hline
\end{tabular}
Beam element is linear elastic and allows transverse shear deformation [9]. Planarity was guaranteed by constraining the out of plane nodes displacements. Mechanical properties are collected in Table 1. Boundary conditions are assumed as in (4-7).

Table 1. Mechanical characteristics of the FEM beam model

Using Lanczos method, the first natural frequency and corresponding free vibration mode shape has been determined. The results show, as expected, that in first

a)

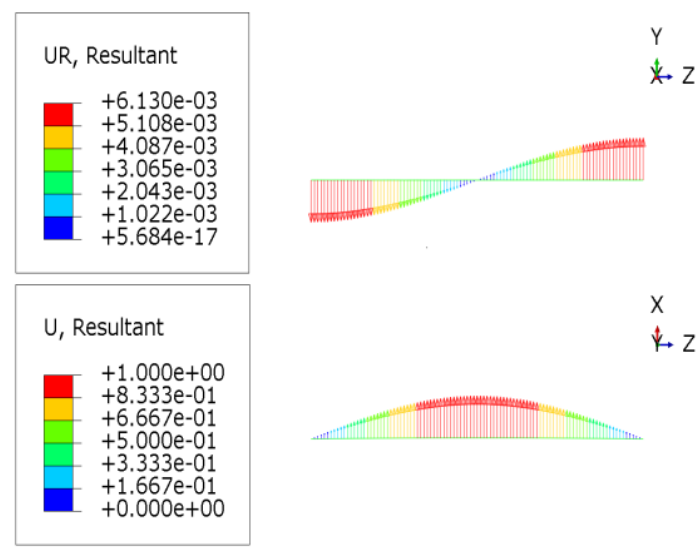

Fig. 3. First natural frequency mode shape, dimensionless vectors of rotation and displacement. 
bending mode we have $W(Z, T)=0$, namely that longitudinal vibrations have no effects on first linear bending shape and natural frequency (Fig. 3). In other words, small amplitudes of transversal vibrations are independent of axial spring stiffness. For all inspected types of axial boundary conditions the first bending linear frequency is $\omega_{0}=461.47 \mathrm{~Hz}(2899.50 \mathrm{rad} / \mathrm{s})$, period $\mathrm{T}=0.002167 \mathrm{sec}$.

Dynamic explicit calculations have been performed to study nonlinear vibrations. External excitation has been modeled as concentrated force in $Z=L / 2$ of the beam in transversal direction, mathematically described by the Dirac delta function $P_{v}=p_{v} \delta\left(Z-\frac{L}{2}\right)$. Amplitude $p_{v}$ is constant (see Tab. 1 for its value) and corresponds to $1 \mathrm{~mm}$ static deflection of middle point of the hingedsimply supported beam, which is equals $2 \%$ its thickness.

In order to get the complete frequency response curve, i.e. how the amplitude of the response varies by

a)

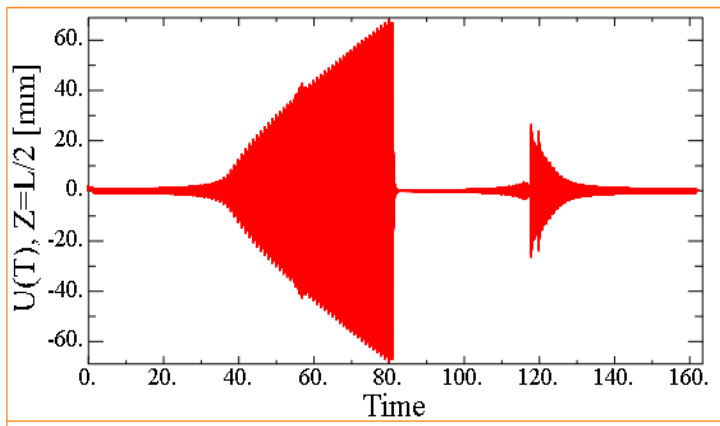

b)

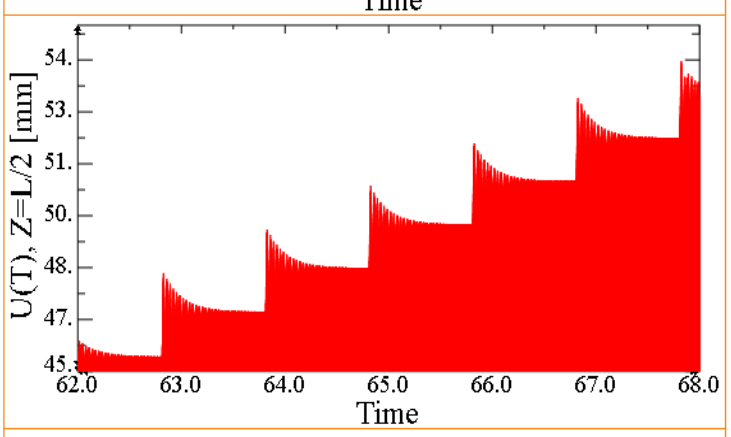

c)

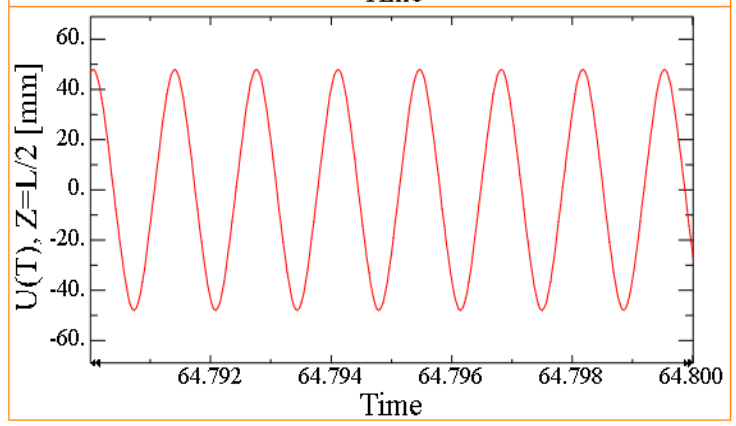

Fig. 4. Time histories of vibrations of hinged-supported-spring $\kappa=4$, reported in three different time scales: (a) global sweep forward from $\frac{\omega}{\omega_{0}}=0$ to $\frac{\omega}{\omega_{0}}=2$, reset $\frac{\omega}{\omega_{0}}=0$ and backward from $\frac{\omega}{\omega_{0}}=2$ to $\frac{\omega}{\omega_{0}}=0$, (b) stabilization of the each frequency from $\frac{\omega}{\omega_{0}}=1.55$ to $\frac{\omega}{\omega_{0}}=1.625$ and (c) time history of a steady-state solution for $\frac{\omega}{\omega_{0}}=1.625$. varying the excitation frequency, forward and backward frequency sweeping are performed. Each excitation frequency $\omega$ has been applied individually, step by step, first with increasing frequency from $0 \mathrm{~Hz}$ to $922.94 \mathrm{~Hz}$ (double of the natural frequency $\omega_{0}$ ). Next, the simulation is reseted and then repeated with decreasing frequency from $922.94 \mathrm{~Hz}$ to $0 \mathrm{~Hz}$. To minimize the computational time a special methodology, similar to a continuation method has been applied, see Tab. 2. This reduced the unwanted jump between the upper and lower branches (when they coexist). The initial conditions to each step have been taken from upshot of the previous step, consistent with the excitation phase.

Table 2. Sampling of excitation frequencies

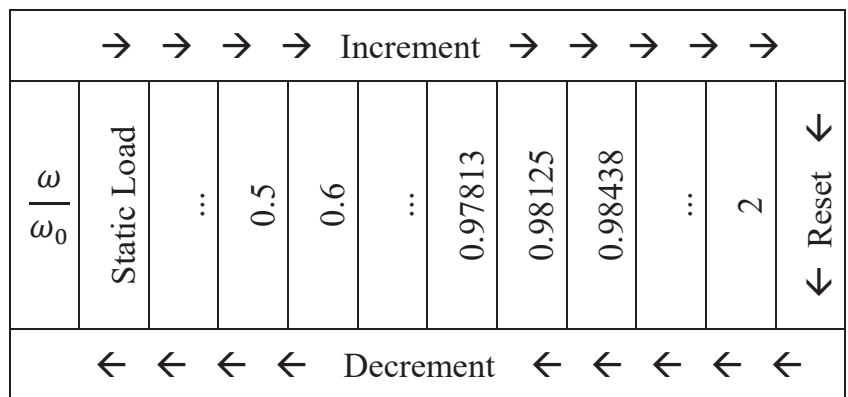

The displacement of the middle point of the beam has been recorded in transversal direction. The time step was constant for each frequency and equal to $\frac{1}{40}$ of excitation period. After a frequency increment/decrement, the simulations run for a sufficient number of periods during which the solution ends its transient and approaches the new attractor (Fig. 4b). The response amplitude is measured at the end of the transient, just before changing again the excitation frequency. An example of a time history obtained with this approach is reported in Fig. 4.

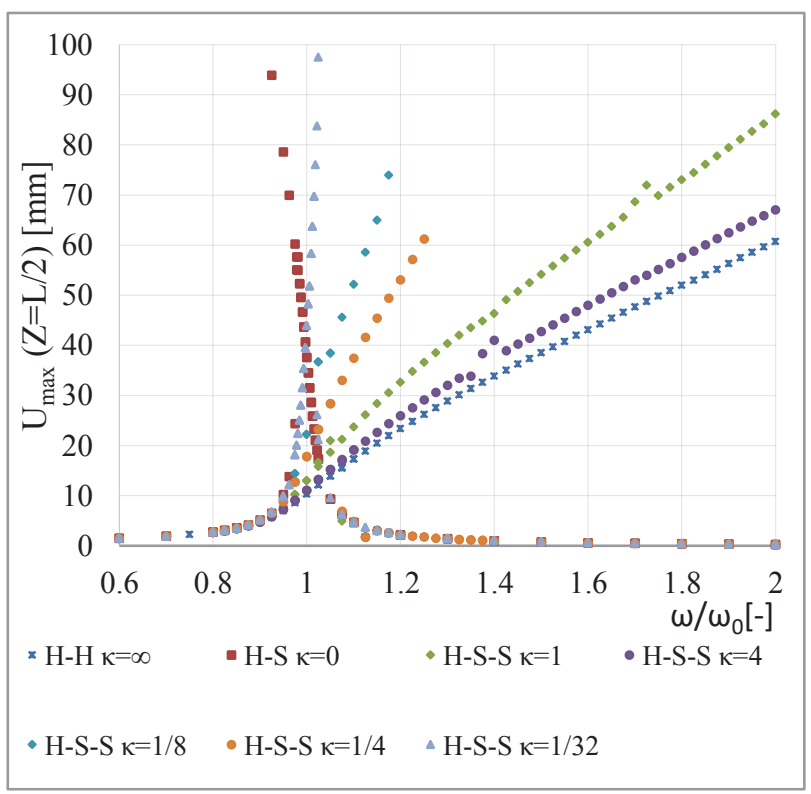

Fig. 5. Frequency response curves of hinged-supported-spring systems, obtained by FEM model, for selected spring stiffness from 0 to $+\infty$. Curves demonstrate effect from softening to hardening, respectively. 
Computations are demanding and very time consuming. Notwithstanding, such an approach allows to study nonlinear systems. Post-processing the many time histories obtained for different values of $\omega$, the final result are the frequency response curves, which are reported in Fig. 5 for different values of $\kappa$.

Jumps from upper branches to lower branches occur for the beam axial stiffness $\kappa$ equal $\frac{1}{8}$ and $\frac{1}{4}$. Moreover, unexpected super or sub harmonic resonances appeared for $\kappa=4$ and $\kappa=1$ for frequencies $\omega / \omega_{0}=1.725$ and $\omega / \omega_{0}=1.40$, respectively. Particular attention has been paid to these zones, and the corresponding time histories are plotted in Fig. 6.

a)

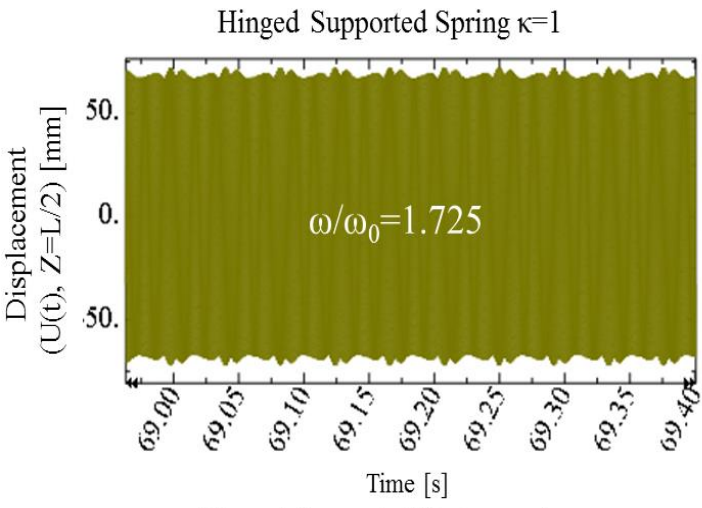

b)

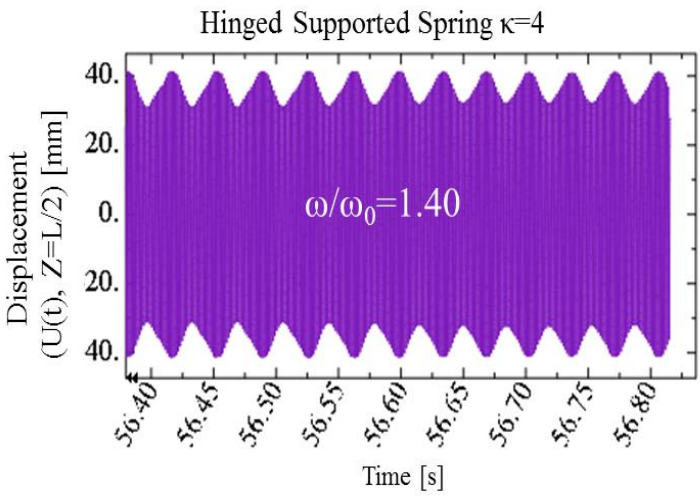

Fig. 6. Time history of superharmonic resonances of hingedsupported-spring (a) $\kappa=1$ and (b) $\kappa=4$.

The Fourier Transform shows relationship between longitudinal and transversal vibrations. In case $\kappa=1$ peaks corresponds to $\omega_{11} \cong 1.725 \omega / \omega_{0}, \quad \omega_{12} \cong$ $5.2 \omega / \omega_{0}, \omega_{13} \cong 6.3 \omega / \omega_{0}$ and for $\kappa=4$ peaks are at $\omega_{41} \cong 1.4 \omega / \omega_{0}$ and $\omega_{42} \cong 4.2 \omega / \omega_{0}$. The ratio between harmonics in both cases are $\omega_{12}=3 \omega_{11}$ and $\omega_{42}=3 \omega_{41}$ (Fig. 7). These cases correspond to internal resonance between the first and the second bending modes.

\section{Conclusions}

The paper presents the results of explicit simulation of damped and forced motion of hinged-supported beam with one end axial spring system. Using the FEM large amplitude vibrations under harmonic excitation are considered. The influence of axial boundary conditions as varying spring stiffness on softening or hardening effect on the frequency response curves is presented. In numerical FEM analyses it has been shown that a 1:3 internal resonances for spring supported cases may occur.

a)

Hinged Supported Beam $\kappa=1$

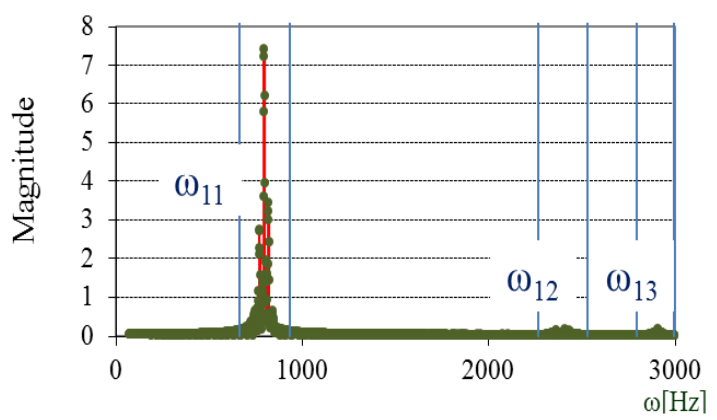

b)

Hinged Supported Beam $\kappa=4$

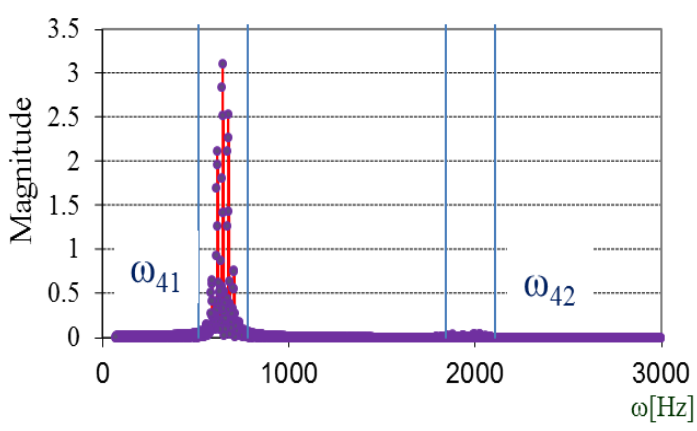

Fig. 7. Fast Fourier transforms of time series of hingedsupported-spring systems (a) $\kappa=1$ and (b) $\kappa=4$.

In further developments the Multiple Time Scales Method will be applied to the mathematical model, and the frequency response curves and their backbone curves will be determined analytically. The proposed analysis can be adapted to any system i.e. nonhomogeneous and with varied cross-section beams, higher modes, different boundary conditions, etc. Other internal resonances will be investigated as well, and for selected cases, the results will be confirmed experimentally.

\section{References}

1. J.N. Reddy, I.R. Singht. Int. J. Num. Mech. Eng. 17(6), p.829-852 (1981)

2. M.R.M Crespo da Silva, C.C. Glynn, J. of Struc. Mech., 6(4), 437-448 (1978)

3. M.R.M Crespo da Silva, C.C. Glynn, J. of Struc. Mech., 6(4), 449-461 (1978)

4. S. Atluri, J. Appl. Mech. 40, 121-126 (1973)

5. G.Rega S. Lenci, Procedia IUTAM 19, 35-42 (2016)

6. S. Lenci, F. Clementi, G. Rega, Meccanina 51(11), 2589-2606, (2016)

7. S. Lenci, G. Rega, Proc. Royal Soc. A, (2016)

8. F. Clementi, S. Lenci, G. Rega, Archive of Appl. Mech. (5)87, 865-880 (2017)

9. Abaqus 6.9 Documentation 